\title{
Letter
}

\section{Experimental Observation of Guanine Tautomers with VUV Photoionization} Jia Zhou, Oleg Kostko, Christophe Nicolas, Xiaonan Tang, Leonid Belau, Mattanjah S. de Vries, and Musahid Ahmed

J. Phys. Chem. A, Article ASAP • DOI: 10.1021/jp811107x • Publication Date (Web): 03 April 2009 Downloaded from http://pubs.acs.org on April 3, 2009

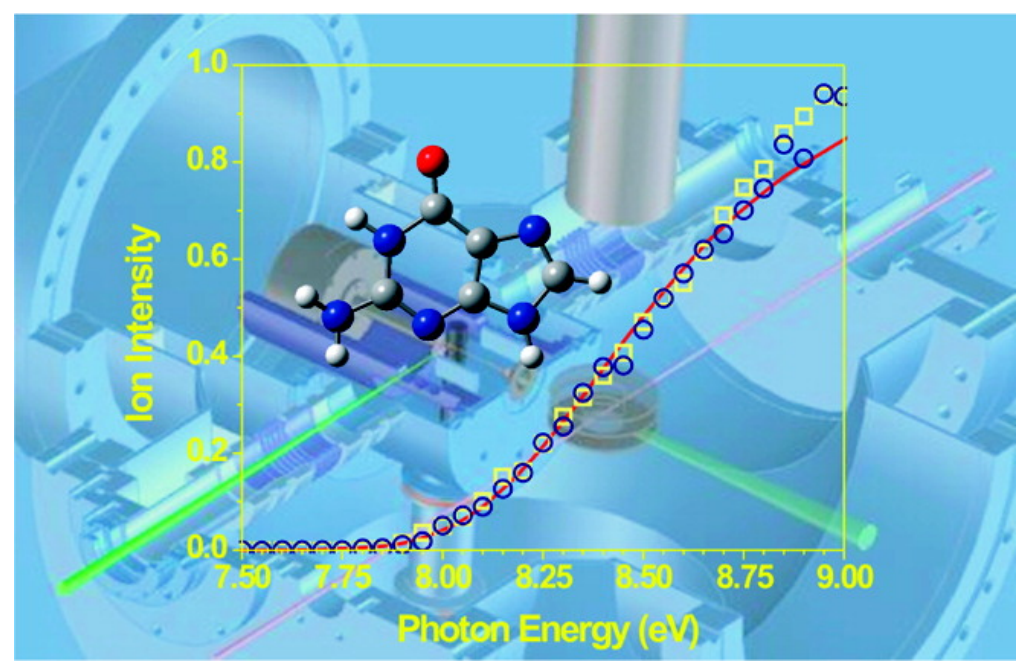

\section{More About This Article}

Additional resources and features associated with this article are available within the HTML version:

- Supporting Information

- Access to high resolution figures

- $\quad$ Links to articles and content related to this article

- Copyright permission to reproduce figures and/or text from this article

\section{View the Full Text HTML}


$\mathbf{X X X X}, x x x, 000$

\title{
Experimental Observation of Guanine Tautomers with VUV Photoionization
}

\author{
Jia Zhou, ${ }^{\dagger}$ Oleg Kostko, ${ }^{\dagger}$ Christophe Nicolas, ${ }^{\dagger}$ Xiaonan Tang,,${ }^{\dagger}$ Leonid Belau, ${ }^{\dagger}$ \\ Mattanjah S. de Vries, ${ }^{*}$ and Musahid Ahmed*, \\ Chemical Sciences Division, Lawrence Berkeley National Laboratory, Berkeley, California 94720, and \\ Department of Chemistry and Biochemistry, University of California, Santa Barbara, California 93106
}

Received: December 16, 2008; Revised Manuscript Received: March 23, 2009

\begin{abstract}
Two methods of preparing guanine in the gas phase, thermal vaporization and laser desorption, have been investigated. The guanine generated by each method is entrained in a molecular beam, single-photon ionized with tunable VUV synchrotron radiation, and analyzed using reflectron mass spectrometry. The recorded photoionization efficiency (PIE) curves show a dramatic difference for experiments performed via thermal vaporization compared to that with laser desorption. The calculated vertical and adiabatic ionization energies for the eight lowest-lying tautomers of guanine suggest that the experimental observations arise from different tautomers being populated in the two different experimental methods.
\end{abstract}

Determinations of ionization energies and other properties of the DNA bases in the gas phase are not trivial. ${ }^{1,2}$ The challenge stems from the fact that transporting these nonvolatile and, in most cases, very fragile molecules to the gas phase leads to extensive fragmentation, making it difficult to generate intact parent molecules. This is further compounded by the possibility of forming different conformers, rotamers, and tautomers during the vaporization process. ${ }^{3}$ For instance, the guanine molecule has four labile hydrogen atoms which can bind to seven possible electronegative binding sites, giving rise to a large number of possible tautomers (Figure 1). According to calculations, ${ }^{4}$ the four lowest-energy tautomers are within $0.1 \mathrm{eV}$ of each other, of which the keto-N9H tautomer (structure shown in Figure 1) is the biologically relevant form. The next low-energy tautomer is approximately $0.2 \mathrm{eV}$ higher in energy, and three more forms are in the $0.26-0.28 \mathrm{eV}$ range; the rest of the tautomers lie above $0.5 \mathrm{eV}$. The eight lowest-lying tautomers and their calculated energies are listed in Table 1.

Traditionally, resonant two-photon ionization (R2PI) and optical double resonance spectroscopies ${ }^{5-9}$ have been used to study the guanine tautomers produced via laser desorption. However, Choi and Miller ${ }^{10}$ showed that the tautomer distribution observed by IR spectroscopy of guanine formed by thermal vaporization with subsequent entrainment in ultracold helium droplets differs from that observed in laser desorption probed by R2PI. This led to a flurry of experimental ${ }^{9}$ and theoretical ${ }^{11,12}$ efforts to reassign the tautomer populations that are formed in the laser desorption process. The proposed explanation for the discrepancy is that the lowest-energy keto tautomers (as well as tautomer 3) have short-lived (picosecond or less) excited states and therefore cannot be detected by nanosecond pulsed R2PI. These tautomers do show up in the helium droplet

* To whom correspondence should be addressed. Address: 6R-2100 Lawrence Berkeley National Laboratory, 1 Cyclotron Road, Berkeley, CA 94720, U.S.A. Phone: (510) 486-6355. Fax: (510) 486-5311. E-mail: MAhmed@lbl.gov.

${ }^{\dagger}$ Lawrence Berkeley National Laboratory.

* University of California.

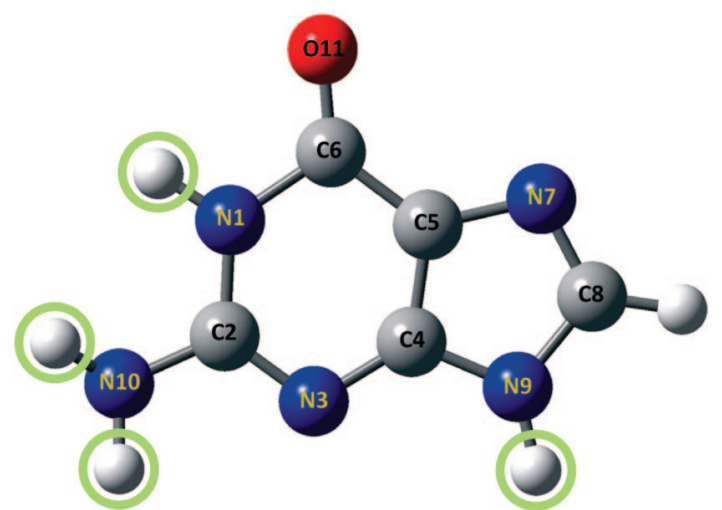

Figure 1. Geometry of the keto-N9H tautomer, the biologically relevant form of guanine. The four labile hydrogen atoms are indicated, and the seven binding sites for them are the five nitrogen atoms and the oxygen atom. To form enol structures, a $\mathrm{H}$ atom migrates from an $\mathrm{NH}$ group to the $\mathrm{O}$ atom.

experiment because it is essentially a ground-state absorption measurement. Thus, it seems that in laser desorption experiments, tautomers $1-8$ are populated in the molecular beam, but of these, only tautomers 3 and 5-7 can be observed by R2PI. In thermal vaporization experiments, where the cooling condition is better, only tautomers $1-4$ are populated, all of which are observed in He droplets. However, as pointed out in a recent review, ${ }^{3}$ comparison of these results is difficult because different experimental detection techniques have been used to assign the tautomer distribution and structure of guanine generated via different gas-phase approaches. Therefore, it is desirable to use a universal detection scheme to directly compare the different vaporization techniques.

Among the four naturally occurring DNA bases, extensive experimental and theoretical studies have shown that guanine in the gas phase has the lowest ionization energy. ${ }^{13,14}$ However, previous single-photon ionization (SPI) studies of guanine have all been conducted utilizing the thermal vaporization method. 
TABLE 1: Computed $\Delta E$, VIE, and AIE for Guanine Tautomers (all energies in $\mathrm{eV})^{a}$

\begin{tabular}{llcccccc}
\hline & & \multicolumn{3}{c}{ this work $^{b}$} & & \multicolumn{2}{c}{ previous work } \\
$N$ & \multicolumn{1}{c}{ tautomer } & $\Delta E$ & $\mathrm{AIE}$ & $\mathrm{VIE}$ & & $\mathrm{VIE}^{c}$ & $\mathrm{VIE}^{d}$ \\
\hline 1 & keto-N7H & 0.000 & 7.80 & 8.16 & & 8.16 & 8.04 \\
2 & keto-N9H & 0.015 & 7.68 & 8.02 & & 8.02 & 7.91 \\
3 & enol-N9H & 0.070 & 7.70 & 8.00 & & 8.00 & 7.89 \\
4 & enol-N9H-trans & 0.098 & 7.75 & 8.02 & & 8.02 & 7.90 \\
5 & enol-N7H & 0.190 & 7.76 & 8.04 & & 8.05 & 7.93 \\
6 & keto-N7H-imino & 0.256 & 8.04 & 8.18 & & 8.18 & 8.07 \\
7 & keto-N7H-imino-cis & 0.267 & 8.12 & 8.25 & & 8.25 & 8.13 \\
8 & keto-N7H-N3H & 0.276 & 8.17 & 8.44 & & 8.44 & 8.33
\end{tabular}

${ }^{a}$ The $N$ refers to the tautomer numbering scheme used in this work in increasing relative energy. AIE and VIE represent adiabatic and vertical ionization energies, with no $\mathrm{ZPE}$ correction. $\Delta E$ is the relative energy including ZPE correction, with respect to the keto $-\mathrm{N} 7 \mathrm{H}$ tautomer. ${ }^{b}$ This work, calculated using B3LYP/6-311+G(d,p). $\quad{ }^{c}$ B3LYP/6-311++G(d,p). ${ }^{21} \quad{ }^{d}$ Restricted Kohn-Sham (B3-LYP functional)/TZVP. ${ }^{11}$

Hush and Cheung, ${ }^{15}$ using photoelectron spectroscopy (PES), reported a vertical ionization energy (VIE) of $8.24 \mathrm{eV}$. Orlov et al. ${ }^{16}$ reported an adiabatic ionization energy (AIE) of 7.77 $\mathrm{eV}$ in a photoionization mass spectrometry measurement. Another PES measurement by Lin et al. ${ }^{17}$ of guanine vaporized at $317^{\circ} \mathrm{C}$ reported a VIE of $8.28 \mathrm{eV}$ with an onset at $7.8 \mathrm{eV}$, in agreement with the earlier results. A recent photoionization mass spectrometry measurement by Belau et al..$^{18}$ of thermally vaporized guanine in a molecular beam in a microhydrated environment reported an appearance energy of $8.1 \pm 0.1 \mathrm{eV}$. Nir et al. report an R2PI ionization energy measurement of 7.905 $\mathrm{eV}$ for the enol-N7H (tautomer 5) form of guanine. ${ }^{7}$ Plekan et al. ${ }^{19}$ performed photofragmentation studies using fixed wavelength VUV radiation and attempted to explain the fragmentation patterns by relating them to published photoelectron spectra.

Cauet et al. ${ }^{13,20}$ and Roca-Sanjuan et al. ${ }^{14}$ discussed various $a b$ initio methods used in calculating the ionization energies for DNA bases with particular emphasis on guanine; they are good reference points for the numerous calculations that have been performed on the photoionization of DNA bases. Cauet et al. ${ }^{13}$ calculated the AIE and VIE of the canonical form of guanine to be 7.82 and $8.27 \mathrm{eV}$, respectively, at the MP2/6$311++\mathrm{G}(\mathrm{d}, \mathrm{p})$ level of theory, while Roca-Sanjuan et al. ${ }^{14}$ reported them as 7.65 and $8.09 \mathrm{eV}$, respectively, using the CASPT2 level of theory. Recently, Shukla and Leszcyzynski, ${ }^{21}$ using the B3LYP/6-311++G(d,p) level of theory, calculated the VIEs for the eight lowest-energy tautomers of guanine, while Marian $^{11}$ used the restricted Kohn-Sham level (B3-LYP functional) to calculate VIEs for the same eight tautomers. Elshakre $^{22}$ and Dolgounitcheva et al. ${ }^{23}$ calculated VIEs for the five-lowest energy tautomers of guanine.

Differences in the ionization energies and the shape of the PIE curve for the various tautomers should be accessible with tunable VUV radiation. To this end, we employed SPI to directly probe the tautomer distribution of guanine formed both via thermal vaporization and via laser desorption with subsequent entrainment in a molecular beam. This method would detect the various tautomers of guanine on an equal footing, allowing for the desired comparison between the two production methods. SPI with tunable VUV radiation, where absorption to intermediate levels is not required, provides a convenient and reliable method to measure ionization energies of fragile biological molecules. ${ }^{24}$ Specifically, threshold photoionization deposits minimal excess energy into a molecule, thereby reducing the fragmentation produced by multiphoton absorption.

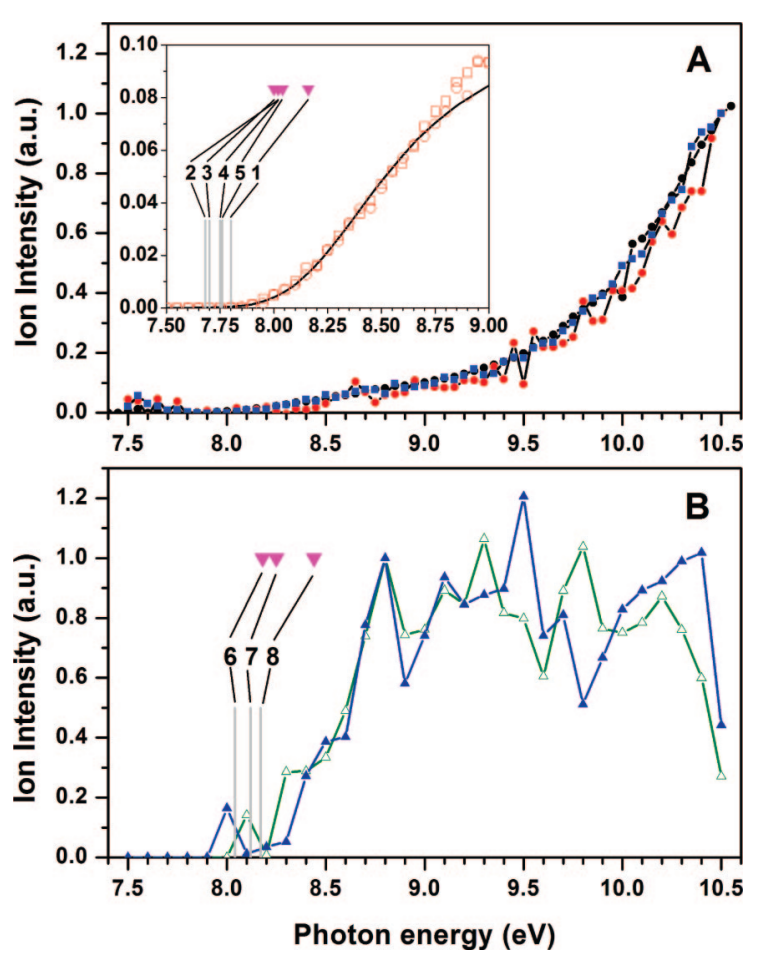

Figure 2. PIE curves for guanine prepared by thermal vaporization (panel A) and laser desorption (panel B). The thermal vaporization experiments in a molecular beam seeded in $\mathrm{Ar}$ are performed at 300 (red -), 325 (blue $\mathbf{\square}$ ), and $395^{\circ} \mathrm{C}$ (black -) with Ar in the gas filter; signal below $7.8 \mathrm{eV}$ arise from second-harmonic light being transmitted through the gas filter. The three thermal vaporization PIEs are normalized to each other at $10.5 \mathrm{eV}$. The inset in panel A shows thermal vaporization data (red $\square$, red $\bigcirc$ ) at $395^{\circ} \mathrm{C}$ with $\mathrm{Kr}$ in the gas filter, together with integrated photoelectron spectrum (solid black line) reported by Lin et al. $\left(317^{\circ} \mathrm{C}\right.$ vaporization cell).$^{17}$ The laser desorption experiments in a molecular beam seeded in $\mathrm{He}\left(\right.$ green $\triangle$ ) and $\mathrm{N}_{2}$ (blue $\boldsymbol{\Delta}$ ) with $\mathrm{Ar}$ in the gas filter are normalized to each other at $8.8 \mathrm{eV}$. The eight lowest-lying tautomers of guanine are denoted by the numbers in energetic order. The gray solid lines represent AIEs, and the (pink $\boldsymbol{\nabla}$ ) represent VIEs calculated in this work and reported in Table 1.

The experiments were performed on a molecular beam apparatus coupled to a $3 \mathrm{~m}$ VUV monochromator on the Chemical Dynamics Beamline at the Advanced Light Source. An in-line gas filter removed higher-order harmonics before the monochromator. ${ }^{25}$ The thermal vaporization source has been described recently in a publication detailing the microhydration of DNA bases. ${ }^{18}$ In the current experiments, the backing pressure was $5.8 \mathrm{psi}$ of Ar through a $100 \mu \mathrm{m}$ diameter nozzle. For the laser desorption experiments, a source that was used to produce carbon clusters ${ }^{26}$ was adapted, for which guanine was pressed with graphite powder to produce a $3 / 8^{\prime \prime}$ rod that was then mounted on the ablation apparatus. A Nd:YLF laser operating at $527 \mathrm{~nm}$ with a repetition rate of $500 \mathrm{~Hz}$ was telescoped with a two lens ( 1 and $0.3 \mathrm{~m}$ plano-convex) combination onto the graphite rod. Backing pressures behind the nozzle were $90(\mathrm{He})$ or $100 \mathrm{psi}\left(\mathrm{N}_{2}\right)$, and the pulsed valve was operated at $500 \mathrm{~Hz}$ to synchronize with the laser. Timeof-flight spectra were recorded for the photoionization energy range between 7.5 and $10.5 \mathrm{eV}$. The typical step size for the PIE scans was $100 \mathrm{meV}$, and the dwell time was $120 \mathrm{~s}$ for the laser desorption experiments and $50 \mathrm{meV}$ and $62.5 \mathrm{~s}$ for thermal vaporization. 
Figure 2 shows the PIE curves for guanine acquired with thermal vaporization at three different temperatures $(300,325$, and $395^{\circ} \mathrm{C}$ ) (Figure $2 \mathrm{~A}$ ) and laser desorption where the carrier gas was either $\mathrm{N}_{2}$ or $\mathrm{He}$ (Figure 2B). The PIE curves from thermal vaporization experiments are very different in shape compared to those from laser desorption experiments. As will be discussed below, different wavelength dependences of the photoionization efficiency for different tautomers are expected. Therefore, the different shapes of the photoionization curves can be correlated with different tautomer distributions. The thermal vaporization PIE curves show a very gentle curvature with an onset at $7.75 \pm 0.05 \mathrm{eV}$, while the laser desorption experiments show a rise in intensity at $8.2 \mathrm{eV}$. The error in the energy scale for the laser desorption experiment is $\pm 0.1 \mathrm{eV}$. The higher signal-to-noise ratio of the thermal vaporization experiments, especially at higher vaporization temperatures (325 and $395{ }^{\circ} \mathrm{C}$ ), is due to the higher guanine density in the supersonic molecular beam together with higher detection repetition rate, $8 \mathrm{KHz}$, as opposed to the $0.5 \mathrm{KHz}$ of the laser desorption experiments. There are also the mechanical complications of a rotating and translating rod coupled to a pulsed valve and laser. Shown in the inset of Figure 2A is a PIE curve derived from integrating the photoelectron (PE) spectrum reported by Lin et al. ${ }^{17}$ It is in agreement with our PIE curves up to $9 \mathrm{eV}$ for the two thermal vaporization data sets recorded at $395{ }^{\circ} \mathrm{C}$ with $\mathrm{Kr}$ in the gas filter. When $\mathrm{Ar}$ is used in the gas filter, second-harmonic radiation is transmitted along with the fundamental below $7.9 \mathrm{eV}$, complicating the determination of ionization onsets. With $\mathrm{Kr}$ in the gas filter, the onset for the thermal vaporization PIE curves measured in this work is 7.75 $\pm 0.05 \mathrm{eV}$, a remarkable agreement with the onset for the PE spectrum at $7.8 \mathrm{eV}$.

A comparison with the PE spectrum reported by Lin et al. ${ }^{17}$ also shows that only the ground electronic state is accessed below $9 \mathrm{eV}$ in the PIE curves. Theoretical work by Dolgounitcheva et al. ${ }^{23}$ confirms that this is at least the case for tautomers 1-5. Therefore, the shapes of the PIE curves up to $9 \mathrm{eV}$ depend mostly on the Franck-Condon overlap of the neutral and cation ground electronic states, which differ for the different tautomers. To aid the interpretation of the PIE spectra, we performed $a b$ initio calculations of the VIEs and AIEs for the eight lowest-lying tautomers of guanine at the B3LYP level with the $6-311+\mathrm{G}(\mathrm{d}, \mathrm{p})$ basis set using Gaussian $03 ;{ }^{27}$ the results are shown in Table 1 . Geometry optimizations were carried out for the neutral and cation ground states of each tautomer, and AIEs were obtained from the differences in the ground-state energies. Zero-point energy (ZPE) corrections showed minimal change $(<0.012 \mathrm{eV})$ in the AIEs. The VIEs were obtained by single-point energy calculations of cations with neutral groundstate geometries. For simplicity, the tautomers are numerically labeled in accordance with their energetic ordering in the neutral state. Also shown in Table 1 are the calculated VIEs of Shukla and Leszczynski ${ }^{21}$ and Marian; ${ }^{11}$ our VIEs are in complete agreement with the former and $0.11-0.12 \mathrm{eV}$ higher compared to the latter.

The four tautomers that were identified in the He droplet experiments are tautomers $1-4$. The calculated AIEs for these tautomers are 7.80, 7.68, 7.70, and $7.75 \mathrm{eV}$, respectively. The measured onset in the PIE curves for the thermal vaporization experiments is $7.75 \mathrm{eV}$, and assuming this onset is adiabatic in nature, the measured AIE is $7.75 \mathrm{eV}$ for thermally vaporized guanine. This is consistent with the presence of the four lowestenergy tautomers (which lie within $0.1 \mathrm{eV}$ of each other) in our experiment, in agreement with the thermal vaporization $\mathrm{He}$
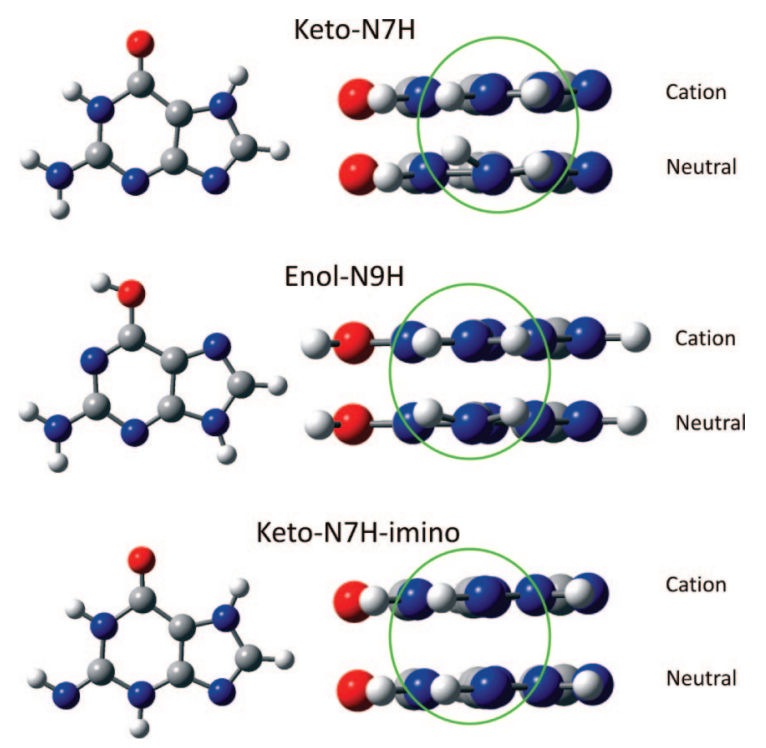

Figure 3. Guanine tautomers 1, 3, and 6, representing the geometry change upon ionization for each class of tautomers. Notice that for the tautomers without an $\mathrm{NH}_{2}$ group, the molecular structures are essentially planar in both the neutral and cation ground states, whereas for the tautomers with an $\mathrm{NH}_{2}$ group, the hydrogen atoms of the $\mathrm{NH}_{2}$ group are out of plane in the neutral ground state.

droplet experiments. ${ }^{10}$ The vaporization temperatures in the current experiments were in the range of $300-395{ }^{\circ} \mathrm{C}$, while the $\mathrm{He}$ droplet experiments were performed at $350{ }^{\circ} \mathrm{C}$. In addition to the four tautomers seen in the helium droplet experiments, tautomer 5, which lies $0.19 \mathrm{eV}$ above tautomer 1 with a calculated AIE of $7.76 \mathrm{eV}$, could also be populated in the thermal experiments. The VIEs calculated for tautomers $1-5$ are $8.16,8.02,8.00,8.02$, and $8.04 \mathrm{eV}$, respectively, which gives a difference of $0.36,0.34,0.30,0.27$, and $0.28 \mathrm{eV}$ from the calculated AIEs. This difference in the ionization energies, especially for the two lowest-energy tautomers, has been observed before in theoretical calculations ${ }^{13,14,20}$ and has been commented upon. It suggests a poor Franck-Condon overlap between the neutral and cation potential energy surfaces, which would lead to a slow onset in the PIE curve. This is indeed observed in the experimental PIE for the thermal vaporization experiments. The neutral and cation geometries of three representative tautomers are shown in Figure 3. It is readily apparent that the $\mathrm{NH}_{2}$ (amino) moiety undergoes a significant geometry change when it is ionized, becoming coplanar with the ring for the five lowest-energy tautomers. This change in geometry would give rise to the gentle curvature observed in the PIE curves.

Now, we turn our attention to the three tautomers that lie approximately $0.25 \mathrm{eV}$ above tautomer 1 , which were observed in the previously published laser desorption molecular beam experiments in addition to tautomer 5 and to a lesser extent $4 .^{3}$ The PIE curves shown in Figure $2 \mathrm{~B}$ for the laser-desorbed guanine are very different from those in the thermal case. There is a rise in signal at $8.2 \mathrm{eV}$. This onset in ionization within the large error limits $( \pm 0.1)$ agrees well with the AIEs calculated for tautomers 6 and 7, which are 8.04 and $8.12 \mathrm{eV}$, respectively. Furthermore, with the absence of the amino moiety in these two tautomers, there is only minor geometry change upon ionization, which is reflected in the calculated VIEs, 8.18 and $8.25 \mathrm{eV}$. The difference between the VIE and AIE for these tautomers is 0.13 and $0.14 \mathrm{eV}$, respectively, which is half of that for the lower-energy tautomers 1-5. Therefore, the Franck-Condon overlap between the neutral and cation ground- 
state potential energy surfaces should be considerably better, resulting in a sharper rise in the PIE, consistent with the experimental observation. Tautomer 8 could also be populated in the laser desorption experiments with calculated AIEs and VIEs of 8.17 and 8.44, respectively. However, with poor Franck-Condon overlap comparable to the low-lying tautomers, its PIE signature would be hidden by the more dominant tautomers 6 and 7 .

There is another noticeable difference in the PIE curves acquired with thermal vaporization and laser desorption. Instead of continuously increasing, as is the trend for the PIE curves from the thermal vaporization experiment, the PIE curves from the laser desorption experiment show a plateau between 8.9 and $10.2 \mathrm{eV}$. After $10.2 \mathrm{eV}$, the ion intensity from the laser desorption experiment actually starts to decrease. There may be several contributing factors causing this difference in the PIE curves at higher energies. As the above discussion suggested, the two methods of preparing guanine may yield different tautomer distributions. Particularly, tautomers 6 and 7, populated by laser desorption, may have significantly different excited electronic state distributions, leading to this difference in the PIE curves. Along a similar line, there may also be more extensive fragmentation occurring for tautomers 6 and 7, leading to a decrease in the molecular ion intensity at higher photon energies. Tautomers 6 and 7 are energetically higher lying; therefore, fragmentation may occur at lower photon energies. Furthermore, their excited electronic states may differ significantly from the lower-lying tautomers, and there may be fragmentation pathways that occur at lower energies. We have observed similar depletion of parent ion signal in the VUV photoionization of the amino acids tryptophan ${ }^{24}$ and histidine. ${ }^{28}$

The picture that emerges from the comparison of PIE curves from thermally vaporized and laser-desorbed guanine is a difference in the tautomer distributions between the two experimental methods. It seems that in thermal desorption experiments, only tautomers $1-4$, and perhaps 5 , are populated, producing gentle PIE onsets due to poor FC overlap associated with a large difference between AIE and VIE. On the other hand, in laser desorption experiments, tautomers 6 and 7 are also present, and because of their better FC overlap, these tautomers dominate the PI signal with a sharp onset. This result would explain why tautomers 6 and 7 are absent in the $\mathrm{He}$ droplet data and present in the laser desorption data. The fact that the lower-energy keto tautomers were not observed in the R2PI data could still be due to the dynamics of the excited neutral states.

An unequivocal experimental determination of the ionization energies of each guanine tautomer will require more sophisticated spectroscopic approaches. Two such methods are being implemented in our group. The first involves utilizing massanalyzed threshold ionization (MATI), demonstrated elegantly by Choi et al. ${ }^{29}$ for the DNA base thymine, and the second is an IR-VUV two-color photoionization scheme ${ }^{30}$ which follows in the spirit of the work performed by de Vries $^{1}$ and Mons. ${ }^{3}$ With both of these schemes and utilizing VUV ionization, it will be possible to bypass the intermediate excited states of the neutral guanine molecule and to directly probe their photoionization dynamics.

Acknowledgment. This work was supported by the Director, Office of Energy Research, Office of Basic Energy Sciences, Chemical Sciences Division of the U.S. Department of Energy under Contract No. DE-AC02-05CH11231 and, in part, by the National Science Foundation under Grant No. CHE-0615401. M.A. acknowledges support from the Laboratory Directed Research and Development (LDRD) program at LBNL.

\section{References and Notes}

(1) de Vries, M. Radiation Induced Molecular Phenomena in Nucleic Acids; Shukla, M. K., Leszczynski, J., Eds.; Springer: New York, 2008; p 323.

(2) de Vries, M. S.; Hobza, P. Annu. Rev. Phys. Chem. 2007, 58, 585.

(3) Mons, M.; Dimicoli, I.; Piuzzi, F. Radiation Induced Molecular Phenomenon in Nucleic Acids; Shukla, M. K., Leszczynski, J., Eds.; Springer: New York, 2008; p 343.

(4) Hanus, M.; Ryjacek, F.; Kabelac, M.; Kubar, T.; Bogdan, T. V.; Trygubenko, S. A.; Hobza, P. J. Am. Chem. Soc. 2003, 125, 7678.

(5) Nir, E.; Grace, L.; Brauer, B.; de Vries, M. S. J. Am. Chem. Soc. 1999, 121, 4896

(6) Nir, E.; Janzen, C.; Imhof, P.; Kleinermanns, K.; de Vries, M. S. J. Chem. Phys. 2001, 115, 4604.

(7) Nir, E.; Plutzer, C.; Kleinermanns, K.; de Vries, M. Eur. Phys. J. D 2002, 20, 317

(8) Mons, M.; Dimicoli, I.; Piuzzi, F.; Tardivel, B.; Elhanine, M. J. Phys. Chem. A 2002, 106, 5088.

(9) Mons, M.; Piuzzi, F.; Dimicoli, I.; Gorb, L.; Leszczynski, J. J. Phys. Chem. A 2006, 110, 10921.

(10) Choi, M. Y.; Miller, R. E. J. Am. Chem. Soc. 2006, 128, 7320.

(11) Marian, C. M. J. Phys. Chem. A 2007, 111, 1545.

(12) Chen, H.; Li, S. H. J. Phys. Chem. A 2006, 110, 12360.

(13) Cauet, E.; Dehareng, D.; Lievin, J. J. Phys. Chem. A 2006, 110, 9200

(14) Roca-Sanjuan, D.; Rubio, M.; Merchan, M.; Serrano-Andres, L. J. Chem. Phys. 2006, 125, 084302.

(15) Hush, N. S.; Cheung, A. S. Chem. Phys. Lett. 1975, 34, 11.

(16) Orlov, V. M.; Smirnov, A. N.; Varshavsky, Y. M. Tetrahedron Lett. 1976, 48, 4377.

(17) Lin, J.; Yu, C.; Peng, S.; Akiyama, I.; Li, K.; Lee, L. K.; Lebreton,

P. R. J. Phys. Chem. 1980, 84, 1006.

(18) Belau, L.; Wilson, K. R.; Leone, S. R.; Ahmed, M. J. Phys. Chem. A 2007, 111, 7562.

(19) Plekan, O.; Feyer, V.; Richter, R.; Coreno, M.; de Simone, M.; Prince, K. C. Chem. Phys. 2007, 334, 53.

(20) Cauet, E.; Lievin, J. Advances in Quantum Chemistry; Elsevier Academic Press Inc: San Diego, CA, 2007; Vol. 52, p 121,

(21) Shukla, M. K.; Leszczynski, J. Chem. Phys. Lett. 2006, 429, 261.

(22) Elshakre, M. Int. J. Quantum Chem. 2005, 104, 1.

(23) Dolgounitcheva, O.; Zakrzewski, V. G.; Ortiz, J. V. J. Am. Chem. Soc. 2000, 122, 12304.

(24) Wilson, K. R.; Jimenez-Cruz, M.; Nicolas, C.; Belau, L.; Leone, S. R.; Ahmed, M. J. Phys. Chem. A 2006, 110, 2106.

(25) Suits, A. G.; Heimann, P.; Yang, X. M.; Evans, M.; Hsu, C. W.; Lu, K. T.; Lee, Y. T.; Kung, A. H. Rev. Sci. Instrum. 1995, 66, 4841.

(26) Nicolas, C.; Shu, J. N.; Peterka, D. S.; Hochlaf, M.; Poisson, L.; Leone, S. R.; Ahmed, M. J. Am. Chem. Soc. 2006, 128, 220.

(27) Frisch, M. J.; Trucks, G. W.; Schlegel, H. B.; Scuseria, G. E.; Robb, M. A.; Cheeseman, J. R.; Montgomery, J. A., Jr.; Vreven, T.; Kudin, K. N.; Burant, J. C.; Millam, J. M.; Iyengar, S. S.; Tomasi, J.; Barone, V.; Mennucci, B.; Cossi, M.; Scalmani, G.; Rega, N.; Petersson, G. A.; Nakatsuji, H.; Hada, M.; Ehara, M.; Toyota, K.; Fukuda, R.; Hasegawa, J.; Ishida, M.; Nakajima, T.; Honda, Y.; Kitao, O.; Nakai, H.; Klene, M.; Li, X.; Knox, J. E.; Hratchian, H. P.; Cross, J. B.; Bakken, V.; Adamo, C.; Jaramillo, J.; Gomperts, R.; Stratmann, R. E.; Yazyev, O.; Austin, A. J.; Cammi, R.; Pomelli, C.; Ochterski, J. W.; Ayala, P. Y.; Morokuma, K.; Voth, G. A.; Salvador, P.; Dannenberg, J. J.; Zakrzewski, V. G.; Dapprich, S.; Daniels, A. D.; Strain, M. C.; Farkas, O.; Malick, D. K.; Rabuck, A. D.; Raghavachari, K.; Foresman, J. B.; Ortiz, J. V.; Cui, Q.; Baboul, A. G.; Clifford, S.; Cioslowski, J.; Stefanov, B. B.; Liu, G.; Liashenko, A.; Piskorz, P.; Komaromi, I.; Martin, R. L.; Fox, D. J.; Keith, T.; Al-Laham, M. A.; Peng, C. Y.; Nanayakkara, A.; Challacombe, M.; Gill, P. M. W.; Johnson, B.; Chen, W.; Wong, M. W.; Gonzalez, C.; Pople, J. A. Gaussian 03; Gaussian, Inc.: Pittsburgh, PA, 2004.

(28) Wilson, K. R.; Belau, L.; Nicolas, C.; Jimenez-Cruz, M.; Leone, S. R.; Ahmed, M. Int. J. Mass Spectrom. 2006, 249, 155.

(29) Choi, K. W.; Lee, J. H.; Kim, S. K. J. Am. Chem. Soc. 2005, 127, 15674.

(30) Qian, X. M.; Zhang, T.; Ng, C. Y.; Kung, A. H.; Ahmed, M. Rev. Sci. Instrum. 2003, 74, 2784.

\section{JP811107X}

